\title{
Pene No Conspicuo (Oculto)
}

\section{Inconspicuous Penis}

\author{
Nicolás Fernández ${ }^{1,2,3}$ Angie Katherine Puerto Niño ${ }^{1} \quad$ Juan David Iregui Parra ${ }^{4}$ \\ Jaime Francisco Pérez Niño ${ }^{1,2}$
}

${ }^{1}$ Division de Urologia, Hospital Universitario San Ignacio, Pontificia Universidad Javeriana, Bogotá, Colombia

2 Division de Urologia Pediatrica, Fundacion Santa Fe de Bogota, Bogotá, Colombia

${ }^{3}$ Division of Urology, Hospital for SickKids. University of Toronto, Canada

${ }^{4}$ Departamento de Urología Clínica DESA, Pontificia Universidad Javeriana, Bogotá, Colombia

\author{
Address for correspondence Dr. Nicolas Fernández, MD, PhD, \\ Division de Urologia, Hospital Universitario San Ignacio, Pontificia \\ Universidad Javeriana, Bogotá, Colombia \\ (e-mail: Fernandez.j@javeriana.edu.co).
}

\section{Resumen \\ Palabras Clave \\ - enfermedades urológicas \\ - pene \\ - cirugía urogenital \\ - complicaciones}

El término pene no conspicuo (oculto), agrupa las patologías en las que el pene adopta un tamaño aparentemente más pequeño, ya sea porque se oculta bajo el tejido cicatricial en caso del pene atrapado, cuando presenta un pliegue de piel que reduce el ángulo penoescrotal tratándose del pene en vela, o en los casos en los que el pene es cubierto por prepucio redundante produciendo un pene enterrado. Se realiza una revisión de la definición y terminología utilizada a través de la historia, clasificación actual, etiología, diagnóstico y técnica quirúrgica empleada para la corrección del pene no conspicuo (oculto).

Inconspicuous penis is referred conditions where the penis looks apparently small but is truely hidden under scarring tissue or under redundant mucosa or prepubic fat. These are all different pathologies that are surgically treated. Multiple techniques have been described. The aim of the present article is to review the state of the art in management throughout history, current classification, etiology, diagnosis and surgical techniques for correcting this pathology.

\section{Introducción}

El pene no conspicuo (oculto), es una anomalía infrecuente descrita inicialmente por Keyes en 1919, como la ausencia del pene cuando éste no es cubierto correctamente por la piel y se encuentra enterrado bajo el tegumento del abdomen, muslo o escroto. ${ }^{1}$

En 1986 Maizels y col., hicieron la descripción de esa patología basados en el reporte de 7 casos de niños con penes ocultos tras el tejido adiposo suprapúbico y a su vez, una clasificación que incluía términos como pene enterrado, pene en vela, pene atrapado y micropene. Cada uno de ellos con características clínicas específicas y causas independientes. ${ }^{2}$ A partir de la publicación de este artículo empezó un uso indiscriminado de esos términos, lo que generó bastante confusión en la literatura médica. En 1993 en un intento por aclarar esa confusión y agrupar los términos descritos previamente, Bergeson y col. publicaron un reporte de caso en el cual

\section{received}

March 7, 2016

accepted

February 8, 2018

published online

October 15, 2018
DOI https://doi.org/

$10.1055 / \mathrm{s}-0038-1656557$.

ISSN 0120-789X.

eISSN 2027-0119.
Copyright (c) 2018, Sociedad Colombiana License terms de Urología. Publicado por Thieme Revinter Publicações Ltda., Rio de Janeiro, Brazil. Todos los derechos reservados. 
redefinieron la clasificación previa incluyendo el pene "diminutivo". 3

En 1994 ÓBrien y col. introdujeron el término megaprepucio congénito (MPC). Es a partir de esa descripción que esa palabra llega a la literatura médica bajo la categoría de pene no conspicuo (oculto). ${ }^{4}$ Alexander y col., proponen en 2010 una nueva clasificación, basándose en las características anatómicas del pene y el prepucio. Si hay un tamaño de falo normal y la posición del glande es infraprepucial, se puede clasificar en pene atrapado cuando la causa es cicatriz poscircuncisión, pene enterrado si la anomalía es MPC, o pene oculto si se origina por excesiva grasa suprapúbica y pobre fijación del eje de la piel. Por otro lado, si la posición del glande es prepucial se clasifica como pene en vela por una inadecuada fusión penoescrotal. ${ }^{5}$

La siguiente tabla constituye una ayuda para el abordaje diagnóstico de esa patología (-Fig. 1).

\section{Clasificación}

Adoptamos los términos descritos por Alexander y col. y realizamos una descripción detallada de las tres entidades más comunes de pene no conspicuo (oculto): el pene atrapado, pene en vela y el pene enterrado.

\section{Pene Atrapado}

Se trata de un pene cuyo cuerpo y glande se encuentran ocultos bajo tejido cicatricial y permanecen embebidos en el escroto y en la grasa prepúbica. ${ }^{6}$ Eso puede suceder después de traumas sin embargo, lo más frecuente es un hallazgo postquirúrgico común en circuncisiones, como lo describe Bergeson en las series del Hospital pediátrico de Phoenix, donde el $42 \%$ de los penes pequeños correspondían a esa categoría. ${ }^{3}$ Otros autores como Williams reportan una incidencia del $26 \%$ de pene atrapado en las circuncisiones realizadas en la unidad de

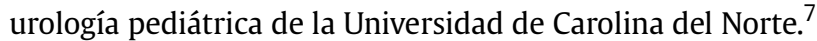

Aquellas circuncisiones en las que se retira muy poco tejido prepucial interno, en donde el borde libre del prepucio protruye más allá de la porción del glande sin nada que lo mantenga aislado, generan un proceso de cicatrización conjunta, que puede progresar a un tejido fibrótico que retrae el pene, dejando visible únicamente el meato uretral en algunas ocasiones. Además, se encuentra en procedimientos que remueven una excesiva cantidad de piel del cuerpo del pene y del prepucio. Por otra parte, puede ocurrir luego de circuncisiones bien realizadas, pero mal indicadas en pacientes con pene oculto por un defecto de fijación del dartos a la piel, o con exceso de grasa prepúbica (-Fig. 2).

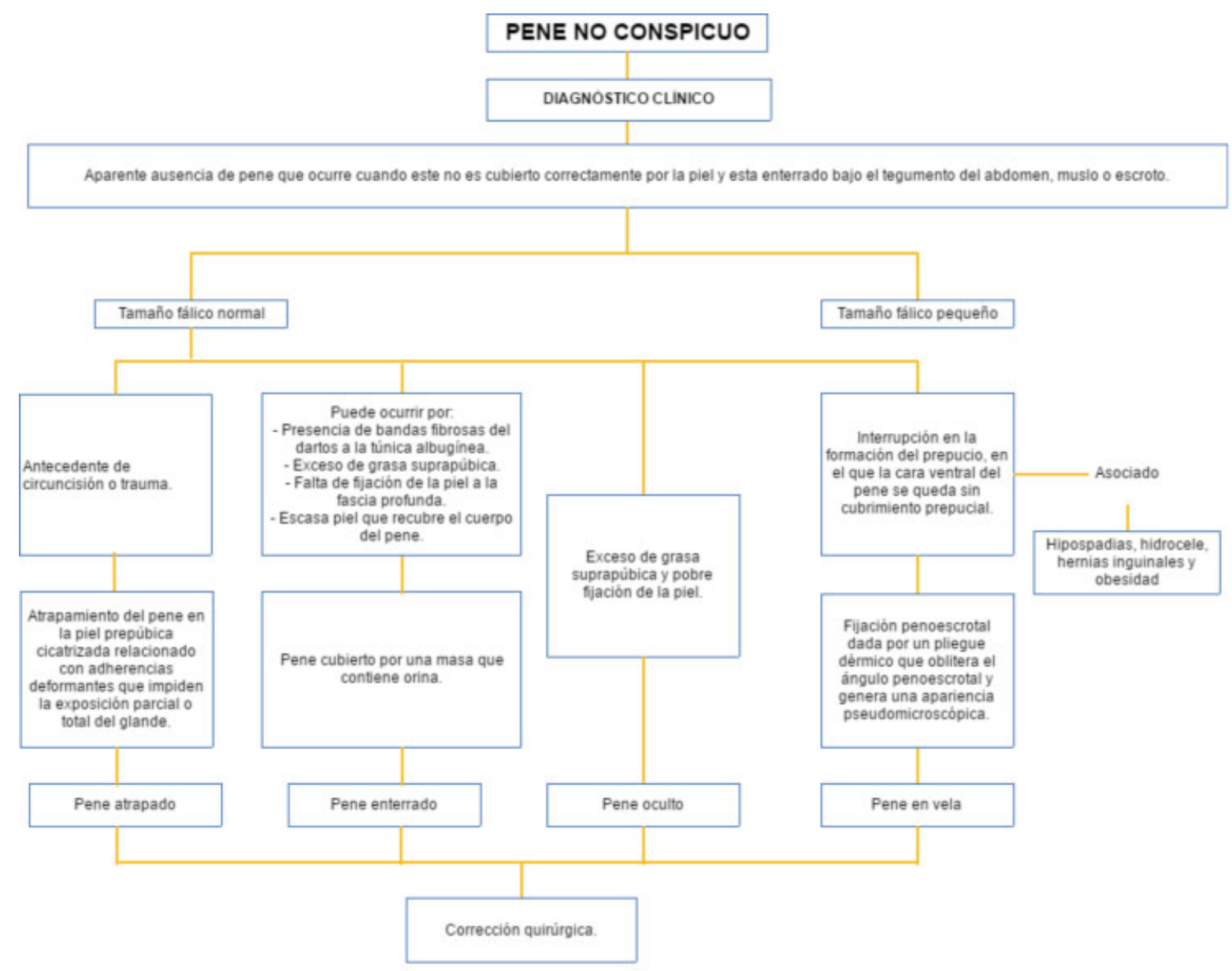

Fig. 1 Abordaje de pene no conspicuo (Adaptado de Alexander y col.). 


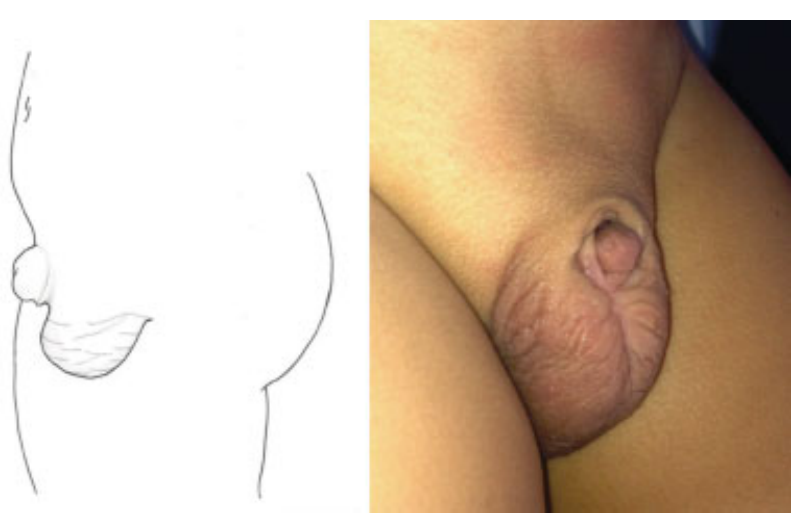

Fig. 2 Representación gráfica de pene atrapado, muestra el cuerpo y glande ocultos bajo tejido cicatricial.

Su diagnóstico es clínico, por lo general con historia de intervención quirúrgica o antecedente de trauma. Al examen físico se evidencia un atrapamiento del eje del pene en la piel prepúbica cicatrizada relacionado con adherencias deformantes que impiden la exposición parcial o total del glande. $^{8}$ No requiere la realización de paraclínicos adicionales y se recomienda su corrección quirúrgica temprana, aunque se han descrito casos con mejoría espontánea con el crecimiento de los pacientes y la llegada de la pubertad. ${ }^{3}$ También está descrito el uso de Betametasona al 0,05\% y retracción manual para el manejo de esa patología, donde el 79\% de 14 pacientes estudiados, lograron reblandecimiento de la cicatriz con fácil exposición del glande. ${ }^{9}$

\section{Corrección Quirúrgica del Pene Atrapado}

Las técnicas usadas para corrección quirúrgica dependen de la severidad de la cicatrización y de la cantidad de tejido sano disponible. Se han descrito Z plastias, liberación de bandas fibróticas, uso de colgajos, injertos de piel de espesor parcial y reparación en dos tiempos. La técnica utilizada por los autores se describe en la - Fig. 3.

La técnica de reparación de dos tiempos sugerida por Ferro, se hace cuando hay deficiencia total de la piel del pene que impide una cobertura adecuada de este. El cuerpo del pene se denuda completamente y se entierra bajo la pared escrotal a lo largo del rafe, con el glande emergiendo desde el fondo del saco escrotal. Después de 6 meses, se realiza una incisión rectangular en la superficie anterior del escroto, con una banda de piel a cada lado del eje, para reconstruir con una sutura mediana. ${ }^{10,11}$ Borsellino y col. encontró una recurrencia del $10 \%$, no asociado a complicaciones. ${ }^{10}$

El trabajo publicado por Lim y col., muestra la experiencia en 36 pacientes con pene oculto después de habérseles practicado circuncisión neonatal, corregidos con la siguiente técnica ( - Fig. 4): inician colocando una sutura de tracción a través del glande, después se realiza una incisión circunferencial de la piel y dartos en el sitio de la circuncisión anterior y una adicional en la línea media ventral de la piel del pene hasta la unión penoescrotal. Posteriormente, se realiza el denudado del pene, liberando el dartos de la fascia de Buck y de la piel, logrando obtener un colgajo dorsal de dartos displásico, el cual es resecado desde la unión penopúbica hasta la cara ventral. La fascia dartos que queda, se sutura a la fascia de Buck en la base del pene utilizando una sutura absorbible. El cubrimiento de piel se
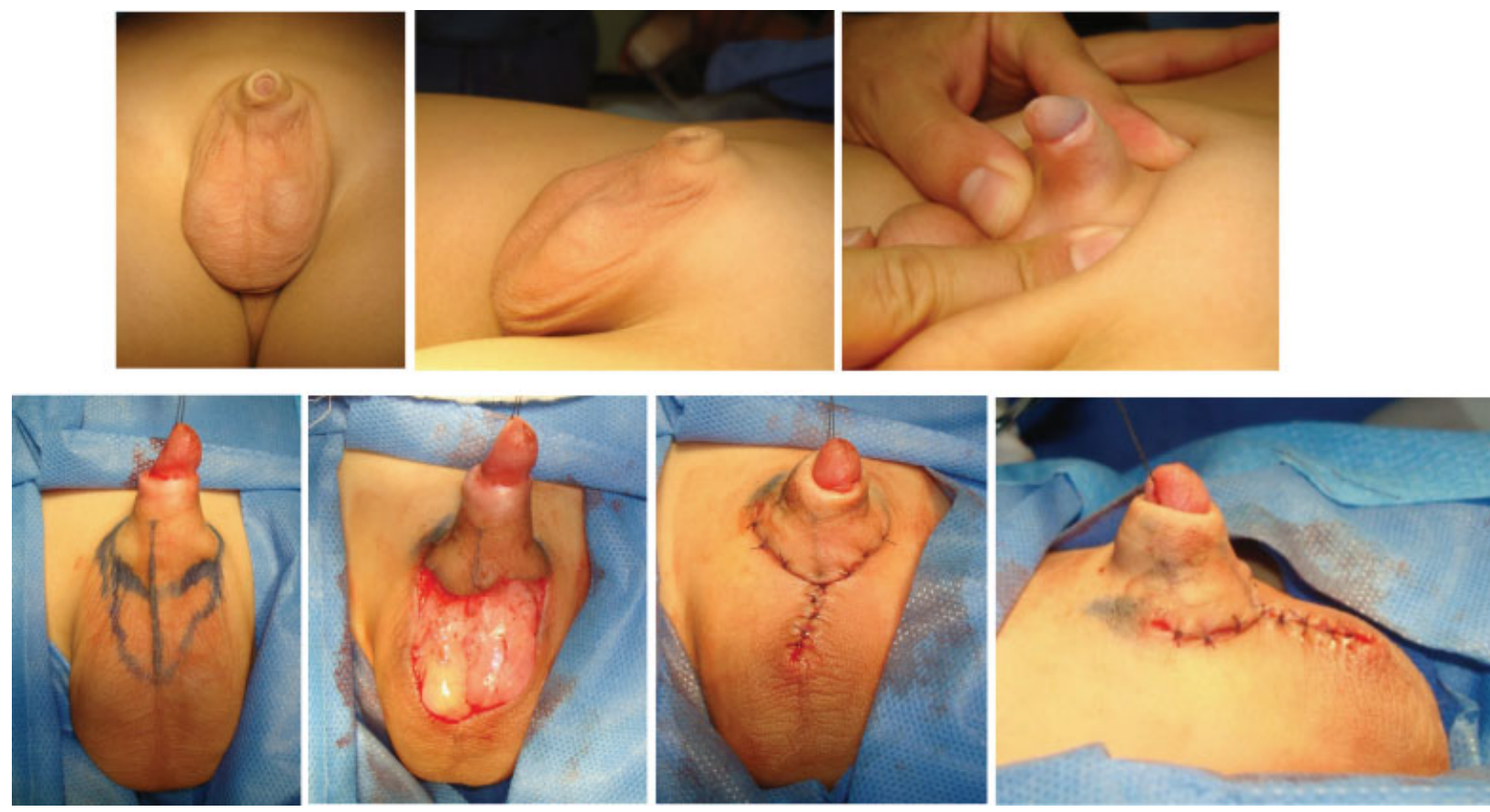

Fig. 3 Técnica empleada por los autores para la corrección del pene atrapado. Nótese en este caso, la deficiente piel ventral con una inserción alta de la unión penoescrotal. Se demarca y realiza circuncisión de la piel en la base del pene con preservación del dartos. Posteriormente, una demarcación ventral en forma de $\mathrm{V}$ con el vértice hacia el aspecto caudal. Se reseca la piel del área comprendida entre esta $\mathrm{V}$ preservando el dartos y posteriormente se suturan los bordes cruentos entre la piel que circuncida el pene y el defecto de piel caudal. 


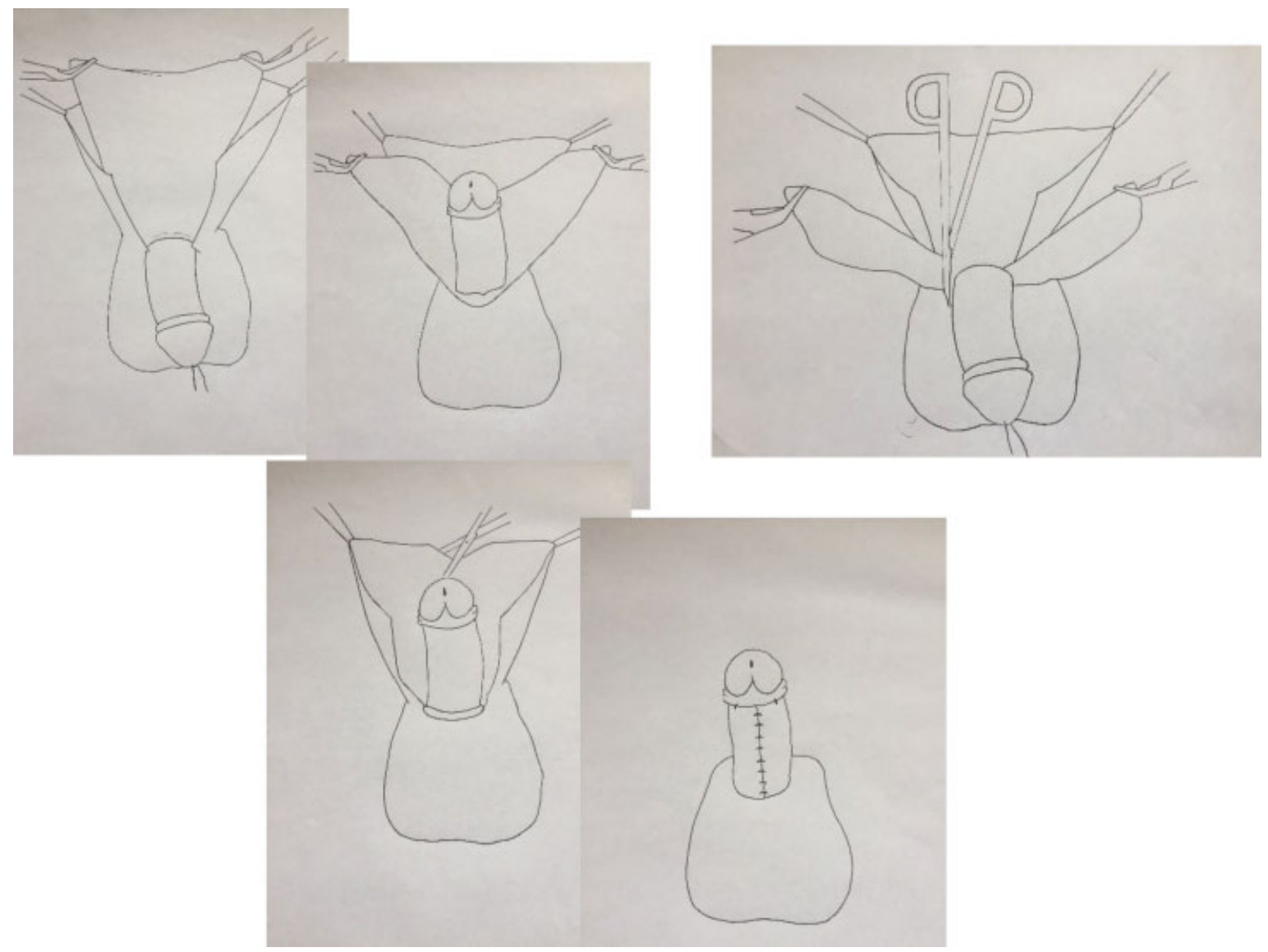

Fig. 4 Corrección quirúrgica del pene atrapado (Adoptado de Lim y col.).

maneja rotando dos colgajos desde dorsal a ventral. Se reportó una complicación, que consistía en la presencia de hematoma el cual resolvió sin secuelas. En cuanto al compromiso neurológico, se encontró hiposensibilidad del glande en un paciente. ${ }^{12}$

\section{Pene en Vela}

Es descrito por primera vez en 1953 en la literatura italiana con el término "virga palmata," y más tarde publicado en la literatura anglosajona por Glanz y col. en 1968 como una condición presente desde el nacimiento en la que existe una red o un pliegue dérmico que reduce el ángulo penoescrotal y genera una apariencia "pseudomicroscópica" del mismo ${ }^{13}$ (- Fig. 5).

La etiología de esa malformación es incierta, se cree que se trata de alguna interrupción en la formación del prepucio, en el que la cara ventral del pene se queda sin cubrimiento prepucial, por lo que el tejido escrotal termina generando su cubrimiento y así se da una obliteración del ángulo. ${ }^{14,15} \mathrm{Su}$ diagnóstico es puramente clínico, teniendo siempre en cuenta que esa condición se puede asociar a hipospadias, hernias inguinales bilaterales, hidroceles y obesidad. ${ }^{3,16-18}$
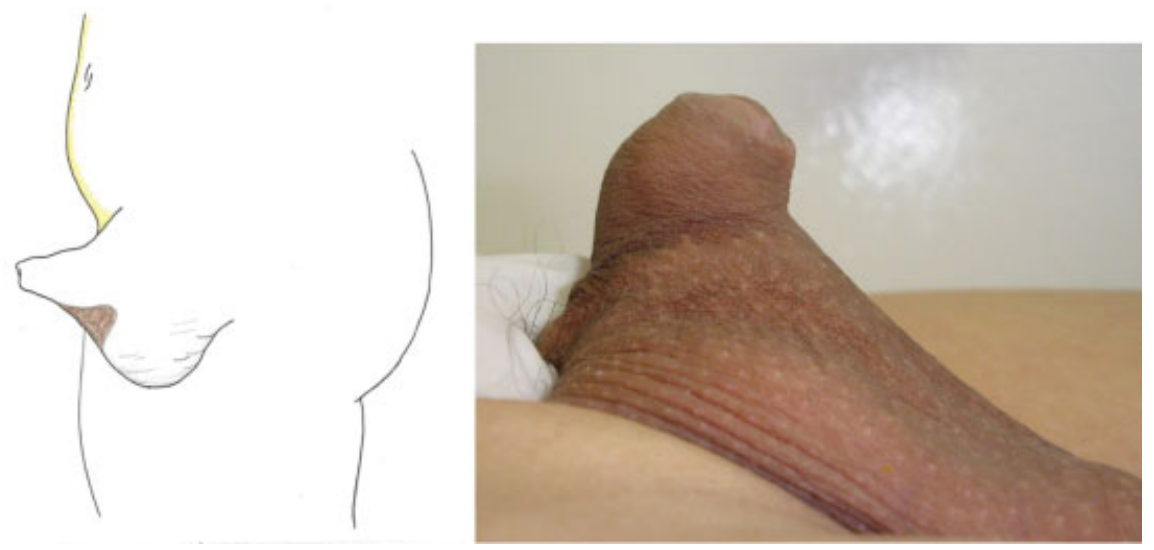

Fig. 5 Pene en vela, muestra la obliteración del ángulo penoescrotal por un pliegue cutáneo que se extiende desde la piel ventral del pene a la pared escrotal. 


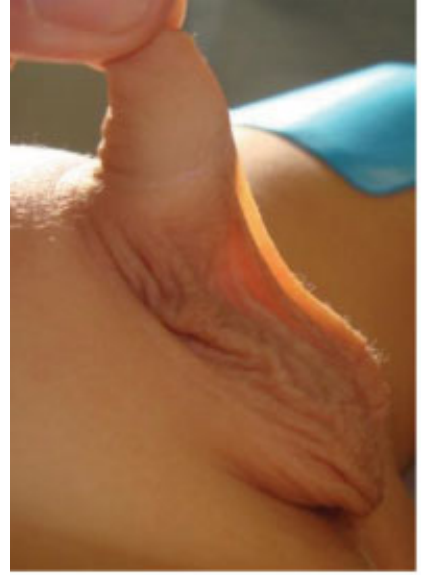

a. Simple grado 1 .

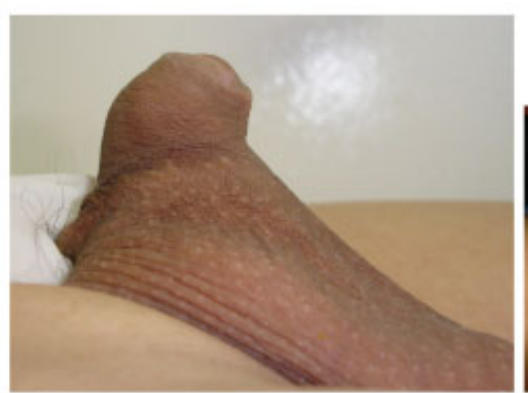

b. Simple grado 2 .

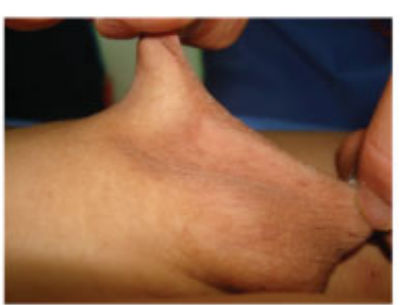

c. Simple grado 3.

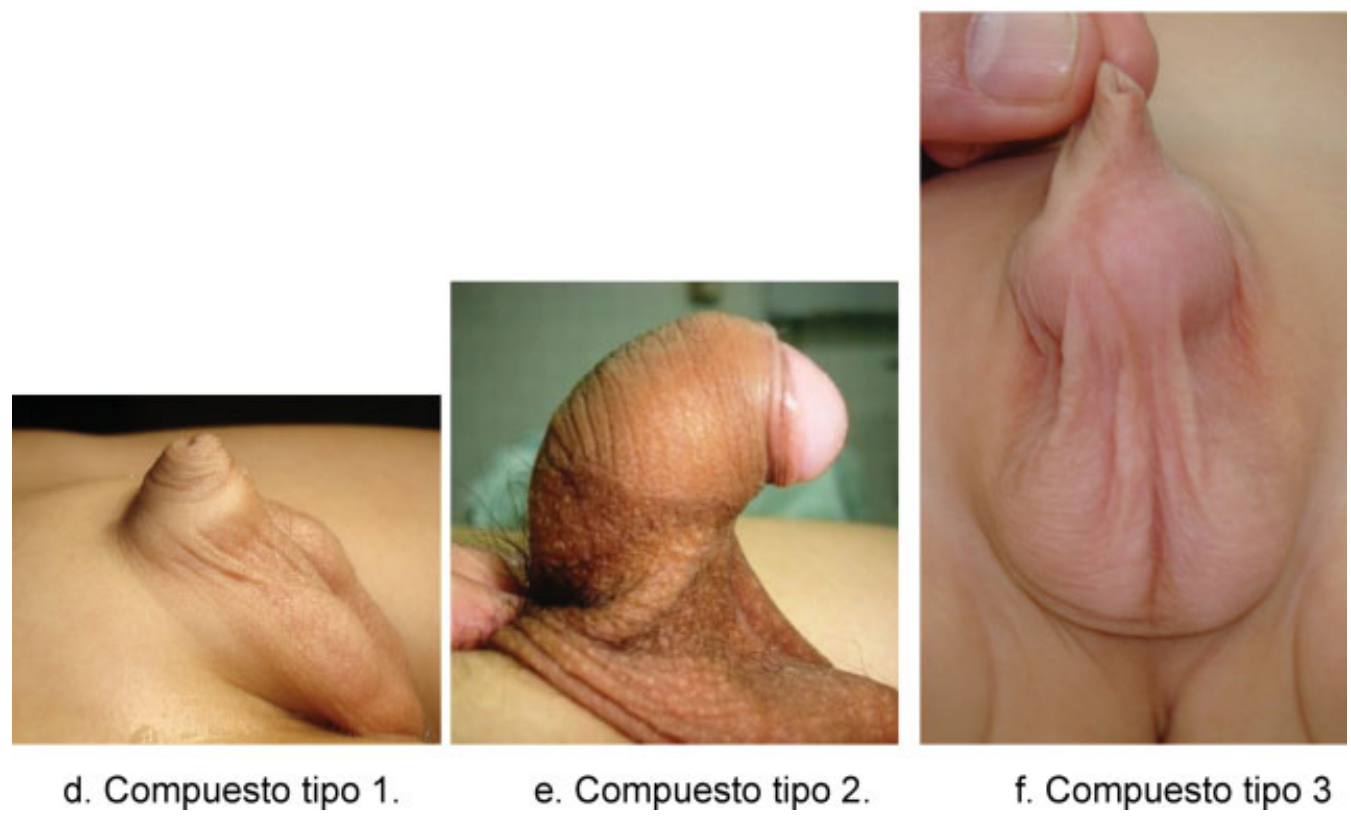

Fig. 6 Clasificación del pene en vela. (a) Simple grado 1. (b) Simple grado 2. (c) Simple grado 3. (d) Compuesto tipo 1. (e) Compuesto tipo 2. (f) Compuesto tipo 3.

La epidemiología del pene en vela no es clara. No existe tanta literatura publicada al respecto, sin embargo, la serie de casos publicados más grande la hizo Montasser y col., de Egipto, quienes por primera vez clasificaron esa condición en causas primarias, secundarias y la extensión de la membrana (-Fig. 6). ${ }^{17}$

Siendo así, el pene en vela primario se compone según Montasser y col., en simple y compuesto, cada uno dividido en tres subclases. El simple tiene 3 grados; el $1^{\circ}$ donde la banda se extiende hasta el $1 / 3$ proximal del eje del pene, en el $2^{\circ}$ la banda se extiende hasta el $1 / 3$ medio del pene y cuando la banda se extiende hasta el $1 / 3$ distal del pene constituye el grado $3^{\circ}$. Así mismo, el pene en vela, primario, compuesto reúne el tipo 1 para aquellos penes que tienen banda con escroto prepeneano, tipo 2 cuando se presenta una banda con curvatura del pene y el tipo 3 con banda amplia. También se incluye, el pene en vela secundario después de circuncisión: pacientes obesos o penes ocultos. ${ }^{17}$ Según los autores, esa clasificación tendría alguna utilidad en la planificación de la corrección quirúrgica y podría evaluar de forma más objetiva los resultados de las diferentes técnicas operatorias; a la fecha no existen nuevas publicaciones que muestren sus ventajas.

\section{Corrección Quirúrgica del Pene en Vela}

Para la corrección quirúrgica del pene en vela primario, Montasser y col., proponen variaciones de la técnica para cada una de las diferentes variedades propuestas por ellos mismos, los objetivos quirúrgicos deben lograr un glande y surco coronal visible, así como una distribución simétrica de la piel dorsal y ventral sin curvaturas y una unión penoescrotal normal. ${ }^{17}$

En términos generales las técnicas de corrección del pene en vela se han caracterizado por tener suturas verticales que cruzan el ángulo penoescrotal, causando en algunos casos contracturas y limitaciones con la erección, por lo que Diley y col., plantea una nueva técnica de corrección en 1999 en la 

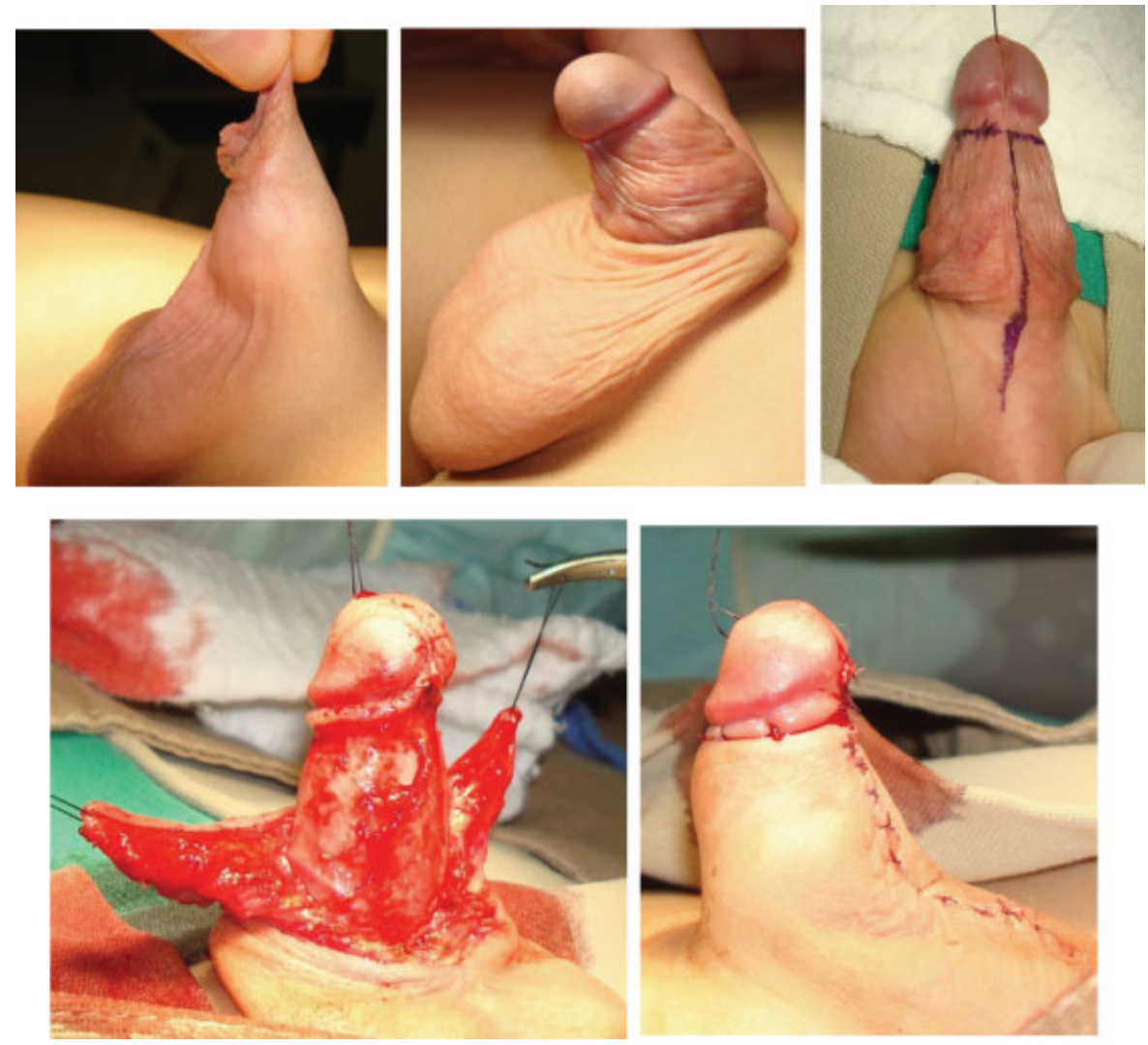

Fig. 7 Técnica empleada por los autores para la corrección del pene en vela. Se hacen dos demarcaciones: una circuncidando la mucosa prepucial aproximadamente a $5 \mathrm{~mm}$ del surco balanoprepucial y una segunda en sentido longitudinal sobre la cara ventral del pene hasta la unión penoescrotal. Se realiza liberación del dartos desde la fascia de Buck llegando hasta la unión penopúbica en la cara dorsal del pene y hasta la unión penoescrotal en la cara ventral. Luego, se reseca el dartos redundante y displásico, se rotan colgajos de piel con técnica de Byers desde el aspecto dorsal hacia el ventral, se reseca la piel redundante de esos colgajos, se fija el dartos a la unión penopúbica y a la unión penoescrotal y se hace el afrontamiento mucocutáneo en la corona del pene y del aspecto ventral de los dos colgajos rotados.

que se divide la membrana en triángulos que componen un rombo, siguiendo el principio de una $Z$ plastias. ${ }^{15}$ La técnica utilizada por los autores se describe en la - Fig. 7.

R.P. Bonitz publicó en 2016, su experiencia comparando tres diferentes técnicas para corregir esa anomalía en 196 pacientes. Las 3 técnicas comparadas fueron: escrotoplastia de Heineke-Mikulicz (HM), la escrotoplastia VY (VY) y la escrotoplastia $Z(Z)$. A pesar de que la escrotoplastia HM es el procedimiento más sencillo, las tres técnicas pueden realizarse según la preferencia y el nivel de habilidad del cirujano. En cuanto a la tasa de complicaciones, VY demostró una mayor tasa de complicaciones $(7,8 \%)$, comparado con HM $(5,3 \%)$ y Z $(2,9 \%)$. Las complicaciones descritas fueron: sangrado, infección de la herida, dehiscencia de la sutura, cicatriz hipertrófica, crecimiento de la piel en "oreja de perro," protrusión de los tractos de sutura y anclaje recurrente de la piel penoescrotal. ${ }^{19}$

\section{Pene Enterrado}

Se describe como un pene de tamaño y aspecto normal que se encuentra escondido dentro de un gran prepucio redundante en forma de domo en el cual se colecciona la orina. ${ }^{5} \mathrm{La}$ mucosa interna es extensa y redundante y se acompaña de piel prepucial escasa y gruesa (-Fig. 8).
Fue descrito por primera vez en 1994 por ÓBrien en un reporte de caso. Aunque no existen estadísticas claras acerca de la prevalencia del MPC al nacimiento, existen varias series de casos.

La etiología de esa patología no es clara, aunque hay varias hipótesis como la presencia de bandas fibrosas que van desde el dartos a la túnica albugínea, desplazando el pene de su posición normal, ${ }^{20-23}$ el exceso de grasa suprapúbica, 22,24 la escasa piel y su falta de fijación a la fascia profunda, ${ }^{25,26}$ y la presencia de fibras poco desarrolladas e hipotróficas del músculo liso del dartos que se distribuyen aleatoriamente en el tejido subcutáneo, sin configuración paralela. ${ }^{27}$

La presentación clínica de esa entidad, corresponde a un pene cubierto por una masa que contiene orina, la que durante la micción se puede extender hasta el escroto, además pueden referir un goteo constante de orina proveniente de la masa. ${ }^{28} \mathrm{El}$ acúmulo crónico de la orina genera la distensión progresiva del prepucio interno ya mencionado. Con el tiempo, puede tornarse tan importante que puede rechazar el cuerpo y glande hacia el tejido adiposo pre púbico, separándolo de la fascia de Buck. ${ }^{5}$

Algunos autores consideran que el diagnóstico es sólo clínico y que no es necesario realizar ningún otro tipo de evaluación previo a la corrección quirúrgica. La inflamación del prepucio interno y la obstrucción generada por el mismo, pueden generar disuria e infecciones urinarias. ${ }^{16,29}$ 


\section{Corrección Quirúrgica del Pene Enterrado}

No hay consenso en la edad de corrección del MPC, en cuanto a si debe hacerse a temprana edad o en la adolescencia. ${ }^{30-32}$ Algunos autores, como Casale, aseguran que la corrección del MPC debe hacerse en los lactantes mayores, una vez ellos empiecen a caminar y se reduzca el tejido adiposo abdominopúbico. ${ }^{33}$ Mientras que autores como Philip, consideran óptimo realizar la corrección quirúrgica tan pronto como se haga el diagnóstico, para mejorar así tanto el defecto fenotípico como la disuria y las posibles complicaciones obstructivas que ellos acarrean. ${ }^{34}$ Según los padres y los cirujanos, la corrección del MPC es homogéneamente exitosa en lactantes y menos en adolescentes. ${ }^{35}$

Entre las técnicas más usadas se encuentran: varios tipos de z plastias, uso de colgajos del prepucio, sección de los ligamentos peneanos, reconstrucción de los ángulos, resección del prepucio interno con reconstrucción, frenuloplastia más movilización de la mucosa prepucial dorsal hacia la cara ventral y plastias ventrales YV. La técnica utilizada por los autores se describe en la $\boldsymbol{- F i g . ~} \mathbf{9}$.

En el trabajo de Alexander y col., se realizó plastia en V ventral para la corrección del megaprepucio, con los siguientes pasos: estiramiento gradual del agujero prepucial con mosquitos, cuidando siempre la integridad de la mucosa para su uso posterior. Se exterioriza el glande, se realiza una incisión en el prepucio interno circunferencialmente y en la cara ventral se realiza en forma de $\mathrm{V}$ creándose así un colgajo ventral y suturándose. Esa técnica basada en un colgajo triangular de tejido prepucial interno ventral, divide el área estenótica y permite preservar la longitud de la piel. A corto plazo, la satisfacción reportada por los padres fue alta en todos los pacientes, al lograr el aspecto de un pene circuncidado y ausencia de curvatura. Esa intervención presenta buenos resultados cosméticos y se convierte en una alternativa para el tratamiento de esa patología sin embargo, se requieren estudios a largo plazo y comparación con otras técnicas. ${ }^{5}$

Por otra parte, William J y col., reportaron un total de 74 pacientes intervenidos en dos instituciones, siguiendo la siguiente técnica ( - Fig. 10): reducción parcial del megaprepucio y exposición del glande, incisión longitudinal por la cara ventral desde el borde prepucial expuesto hasta el extremo proximal del pene, adyacente a la unión penoescrotal marcando en sentido transversal el anillo de dartos. Posteriormente, continúa la incisión ventral en sentido vertical y se circuncida la mucosa, quedando así dos colgajos pediculados por el aspecto dorsal. Se libera el dartos, especialmente en el anillo localizado en la unión penoescrotal, hasta lograr exponer el pene teniendo como plano de fondo la fascia de Buck. Se debe establecer el sitio de la unión penoescrotal, para lograr la reconstrucción de dicho ángulo por la cara ventral, y el ángulo penopúbico por la cara dorsal, todo eso mediante la fijación del dartos a la fascia de Buck, siguiendo el eje del pene. Luego, con una incisión dorsal del colgajo y rotación de los colgajos mucocutáneos hacia ventral, fijando siempre el dartos a la fascia de Buck, logrando de esta manera aproximarlos en la línea media ventral. Se reseca el tejido redundante y se afrontan en la línea media creando un rafé. Observaron que la principal causa en los pacientes con pene no conspicuo (oculto), es la mala inserción del dartos a la fascia de Buck. A 5 años, muestra resultados excelentes en todos los casos, sin evidencia de retracción del pene o del recubrimiento de la piel. Aunque los autores proponen una escala pre y postoperatoria para clasificar el pene enterrado, ella no fue aplicada a cada paciente. ${ }^{20}$

En el trabajo de Rod J y col., se reportó además del resultado cosmético, el resultado funcional, el cual fue bueno en todos los pacientes. Ellos clasificaron los resultados en muy buenos $44 \%$ de los pacientes, buenos (persistencia de un defecto fenotípico moderado que no requiere intervención quirúrgica) en $48 \%$ de los casos y en $8 \%$ resultado malo (reintervención para la resección de un collar interno prepucial redundante y la persistencia del ocultamiento). Del total de casos con resultados buenos, 36\% presentó leve redundancia del prepucio y $64 \%$ presentó moderada retracción del pene. Adicionalmente, 9\% mostró edema distal del pene que no requirió intervención. ${ }^{36}$

Pérez y col. describen una serie de 23 pacientes con diversas formas de pene no conspicuo (oculto), donde trece fueron llevados a reconstrucción de pene con una misma técnica. Los 10 restantes fueron llevados a corte dorsal de prepucio. El seguimiento osciló entre 3 y 30 meses. Se evaluó el resultado

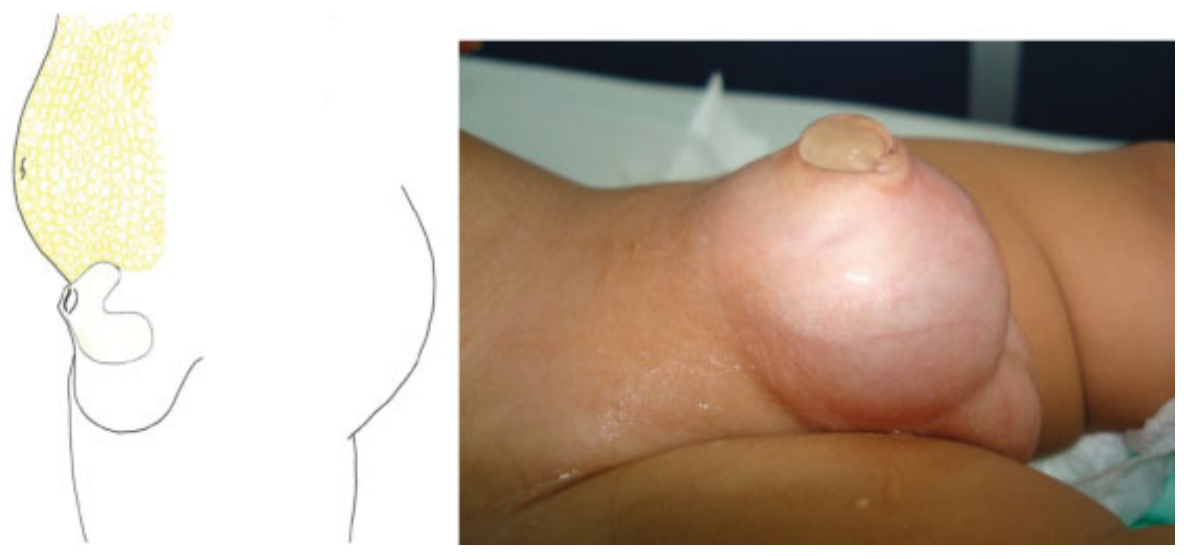

Fig. 8 Pene enterrado, muestra un prepucio redundante que condiciona una cavidad dentro de la que se colecciona orina, asociado a piel escasa y exceso de grasa suprapúbica. 

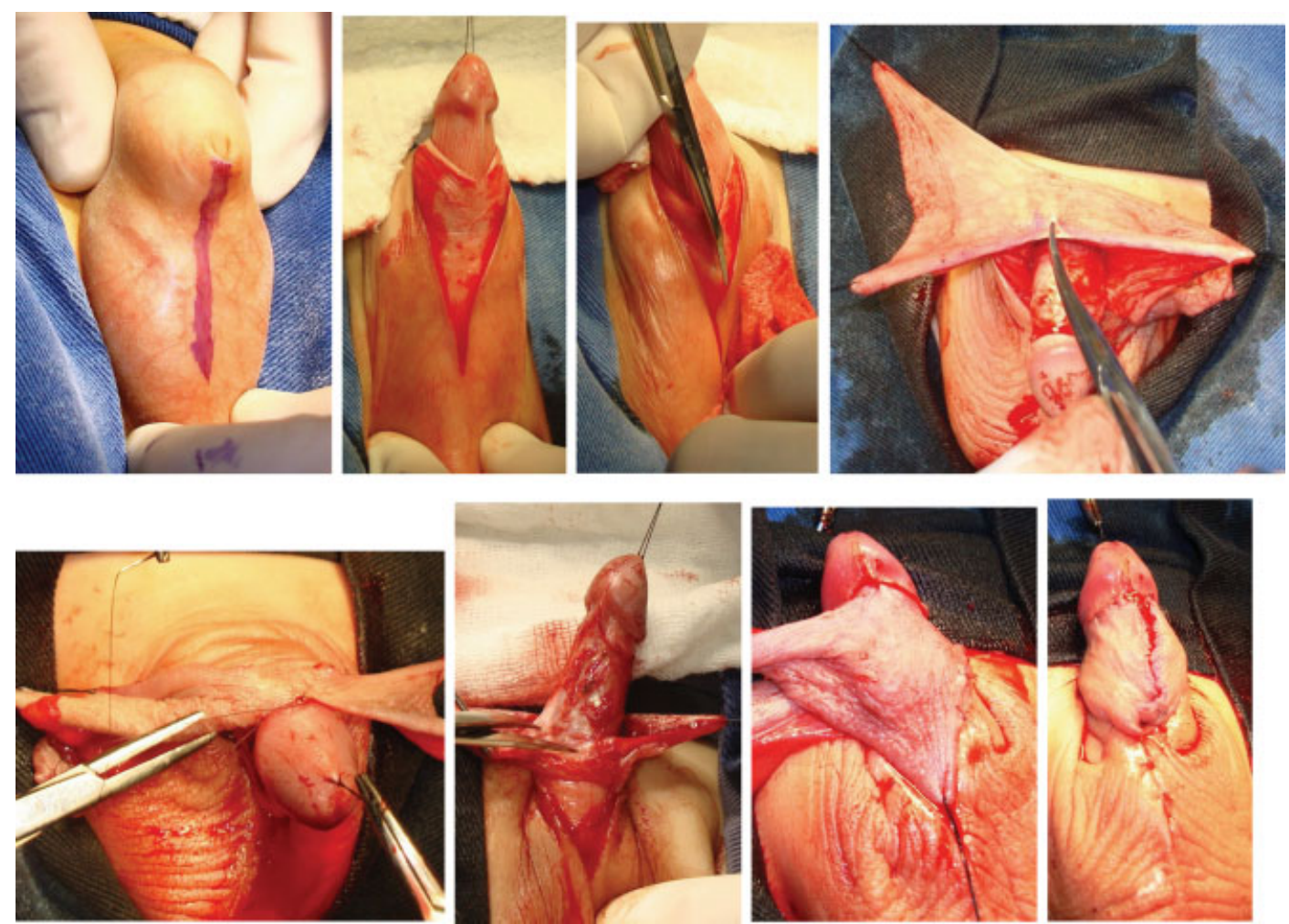

Fig. 9 Técnica empleada por los autores para la corrección del pene enterrado. Teniendo en cuenta que en la gran mayoría de estos penes, no es posible la reducción del anillo fimótico, es necesario hacer un corte longitudinal en la cara ventral del pene desde el aspecto más distal del orificio prepucial hasta alcanzar 1 o $2 \mathrm{~cm}$ por debajo de la unión penoescrotal. Esa incisión permite la liberación del glande. Se coloca punto de reparo para tracción del glande y se prolonga la incisión previa en la mucosa prepucial hasta lograr la liberación completa del pene. Luego se realiza circuncisión de la mucosa prepucial a $5 \mathrm{~mm}$ del surco balanoprepucial. Se realiza liberación del dartos desde la fascia de Buck llegando hasta la unión penopúbica en la cara dorsal del pene y hasta la unión penoescrotal en la cara ventral. Luego, se reseca el dartos redundante y displásico, se rotan los colgajos de piel con la técnica de Byers desde el aspecto dorsal hacia el ventral, se reseca la piel redundante de esos colgajos, se fija el dartos a la unión penopúbica y a la unión penoescrotal y se hace el afrontamiento mucocutáneo en la corona del pene y del aspecto ventral de los dos colgajos rotados.

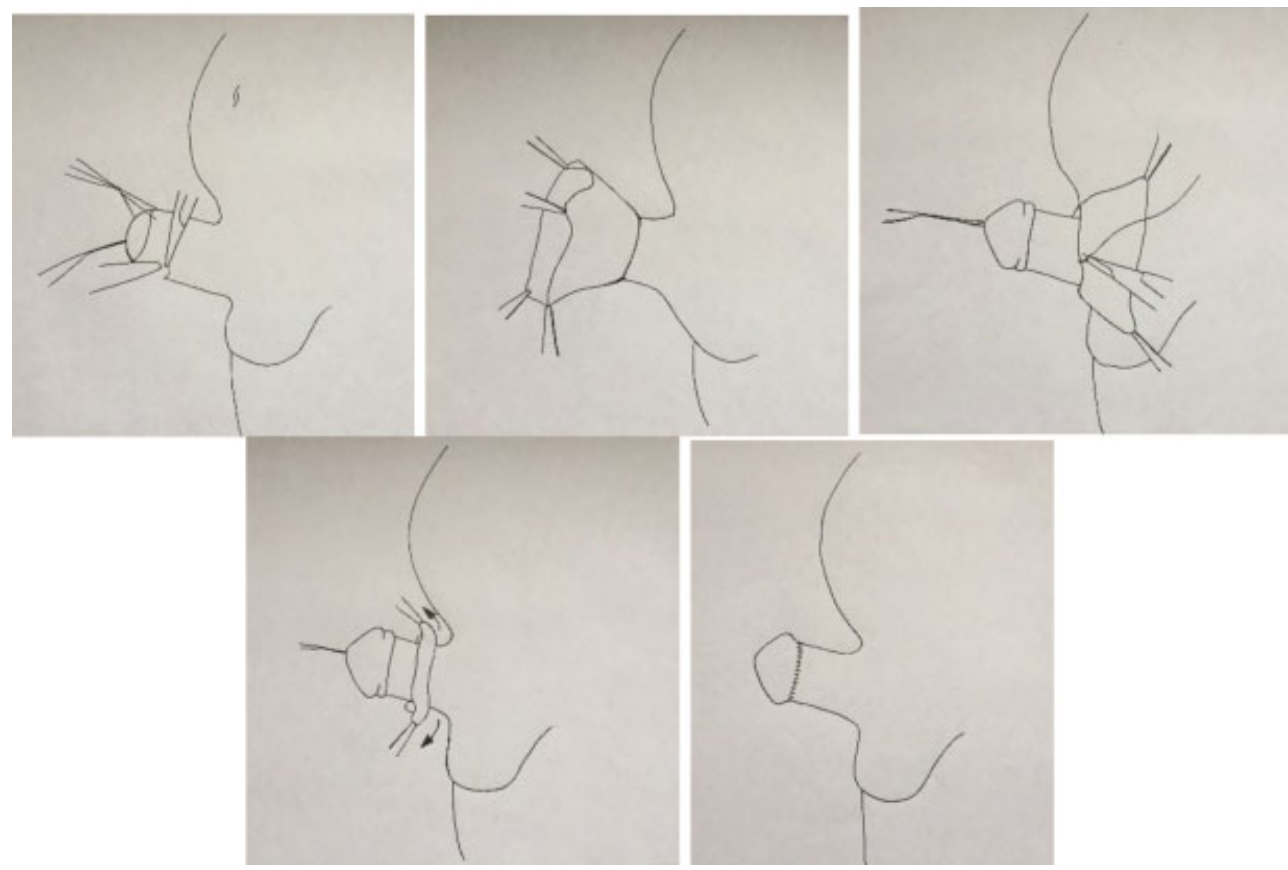

Fig. 10 Corrección quirúrgica del pene enterrado (Adoptado de William J y col.). 
cosmético valorado por los familiares del paciente y alguno de los autores, basados en registros fotográficos, encontrando que en el $82 \%$ de los casos, fue satisfactorio y parcialmente satisfactorio en el $13,04 \%{ }^{37}$

En general, los resultados de la corrección quirúrgica de pene no conspicuo (oculto) reportados en la literatura son adecuados, simples, reproducibles y con un buen resultado del aspecto fenotípico y funcional. ${ }^{17}$ La gran mayoría, siguen los principios de denudamiento completo de la piel del pene, liberación y resección de las adherencias anormales del dartos y reconstrucción de los ángulos penoescrotal y penopúbico, logrando un glande y surco coronal visible, así como una distribución simétrica de la piel dorsal y ventral sin curvaturas. ${ }^{33}$ A pesar de las múltiples técnicas descritas, no existe una que haya demostrado ventajas sobre la otra. ${ }^{5}$

\section{Conclusiones}

El pene no conspicuo (oculto), es un diagnóstico clínico, que representa un reto para el urólogo que deberá reconocer cuidadosamente las variaciones anatómicas de cada caso en particular.

La intervención quirúrgica incluye diferentes técnicas, sin que la literatura establezca una definitiva. La indicación de su corrección, depende de los síntomas, del aspecto fenotípico y de la edad del paciente, existiendo cierta tendencia a que sea temprano en la vida.

Deben estandarizarse escalas para valorar los resultados funcionales y cosméticos de las cirugías reconstructivas del pene, así como homogenizar la clasificación y terminología utilizada.

\section{Referencias}

1 Keyes E. Phimosis-paraphimosis-tumours of the penis. Urol D Applet Co. 1919;67:649

2 Maizels M, Zaontz M, Donovan J, Bushnick PN, Firlit CF. Surgical correction of the buried penis: description of a classification system and a technique to correct the disorder. J Urol 1986;136 (1 Pt 2):268-271

3 Bergeson PS, Hopkin RJ, Bailey RB Jr, McGill LC, Piatt JP. The inconspicuous penis. Pediatrics 1993;92(06):794-799

4 O'Brien A, Shapiro AM, Frank JD. Phimosis or congenital megaprepuce? Br J Urol 1994;73(06):719-720

5 Alexander A, Lorenzo AJ, Salle JLP, Rode H. The Ventral V-plasty: a simple procedure for the reconstruction of a congenital megaprepuce. J Pediatr Surg 2010;45(08):1741-1747

6 Radhakrishnan J, Reyes HM. Penoplasty for buried penis secondary to "radical" circumcision. J Pediatr Surg 1984;19(06):629-631

7 Williams CP, Richardson BG, Bukowski TP. Importance of identifying the inconspicuous penis: prevention of circumcision complications. Urology 2000;56(01):140-142, discussion 142-143

8 Lipszyc E, Pfister C, Liard A, Mitrofanoff P. Surgical treatment of buried penis. Eur J Pediatr Surg 1997;7(05):292-295

9 Palmer JS, Elder JS, Palmer LS. The use of betamethasone to manage the trapped penis following neonatal circumcision. J Urol 2005; 174(4 Pt 2):1577-1578

10 Borsellino A, Spagnoli A, Vallasciani S, Martini L, Ferro F. Surgical approach to concealed penis: technical refinements and outcome. Urology 2007;69(06):1195-1198

11 Ferro F, Spagnoli A, Villa M, Papendieck CM. A salvage surgical solution for recurrent lymphangioma of the prepuce. $\mathrm{Br} \mathrm{J}$ Plast Surg 2005;58(01):97-99
12 Lim DJ, Barraza MA, Stevens PS. Correction of retractile concealed penis. J Urol 1995;153(05):1668-1670

13 Glanz S. Adult congenital penile deformity. Case report. Plast Reconstr Surg 1968;41(06):579-580

14 Perlmutter AD, Chamberlain JW. Webbed penis without chordee. J Urol 1972;107(02):320-321

15 Dilley AV, Currie BG. Webbed penis. Pediatr Surg Int 1999;15 (5-6):447-448

16 Shapiro SR. Surgical treatment of the "buried" penis. Urology 1987;30(06):554-559

17 El-Koutby M, Mohamed Amin G. Webbed penis: A new classification. J Indian Assoc Pediatr Surg 2010;15(02):50-52

18 Redman JF. A technique for the correction of penoscrotal fusion. J Urol 1985;133(03):432-433

19 Bonitz RP, Hanna MK. Correction of congenital penoscrotal webbing in children: A retrospective review of three surgical techniques. J Pediatr Urol 2016;12(03):161.e1-161.e5

20 Cromie WJ, Ritchey ML, Smith RC, Zagaja GP. Anatomical alignment for the correction of buried penis. J Urol 1998;160 (04):1482-1484

21 Liu X, He DW, Hua Y, Zhang DY, Wei GH. Congenital completely buried penis in boys: anatomical basis and surgical technique. BJU Int 2013;112(02):271-275

22 Boemers TML, De Jong TPVM. The surgical correction of buried penis: a new technique. J Urol 1995;154(2 Pt 1):550-552

23 Wollin M, Duffy PG, Malone PS, Ransley PG. Buried penis. A novel approach. Br J Urol 1990;65(01):97-100

24 Alter GJ, Ehrlich RM. A new technique for correction of the hidden penis in children and adults. J Urol 1999;161(02):455-459

25 Shenoy MU, Srinivasan J, Sully L, Rance CH. Buried penis: surgical correction using liposuction and realignment of skin. BJU Int 2000;86(04):527-530

26 Redman JF. Buried penis: congenital syndrome of a short penile shaft and a paucity of penile shaft skin. J Urol 2005;173(05): 1714-1717http://www.sciencedirect.com/science/article/pii/ S0022534705607033[Internet]

27 Spinoit AF, Van Praet C, Groen LA, Van Laecke E, Praet M, Hoebeke P. Congenital penile pathology is associated with abnormal development of the dartos muscle: a prospective study of primary penile surgery at a tertiary referral center. J Urol 2015;193(05):1620-1624

28 Delgado Oliva FJ, Domínguez Hinarejos C, Serrano Durbá S, Estornell Moragues F, Martínez Verduch M, García Ibarra F. Megaprepucio congénito: diagnóstico y manejo terapéutico. Actas Urol Esp 2006;30(10):1038-1042

29 Ferro F, Spagnoli A, Spyridakis I, Atzori P, Martini L, Borsellino A. Surgical approach to the congenital megaprepuce. J Plast Reconstr Aesthet Surg 2006;59(12):1453-1457

30 Brisson P, Patel H, Chan M, Feins N. Penoplasty for buried penis in children: report of 50 cases. J Pediatr Surg 2001;36(03):421-425

31 Metcalfe PD, Rink RC. The concealed penis: management and outcomes. Curr Opin Urol 2005;15(04):268-272

32 Radhakrishnan J, Razzaq A, Manickam K. Concealed penis. Pediatr Surg Int 2002;18(08):668-672

33 Casale AJ, Beck SD, Cain MP, Adams MC, Rink RC. Concealed penis in childhood: a spectrum of etiology and treatment. J Urol 1999; 162(3 Pt 2):1165-1168

34 Philip I, Nicholas JL. Congenital giant prepucial sac: case reports. J Pediatr Surg 1999;34(03):507-508

35 Herndon CDA, Casale AJ, Cain MP, Rink RC. Long-term outcome of the surgical treatment of concealed penis. J Urol 2003;170(4 Pt 2):1695-1697, discussion 1697

36 Rod J, Desmonts A, Petit T, Ravasse P. Congenital megaprepuce: a 12-year experience ( 52 cases) of this specific form of buried penis. J Pediatr Urol 2013;9(6 Pt A):784-788

37 Niño Jaime Francisco P, Parra Juan David I, Díaz Angeline R, Trujillo Nicolás V, Bonilla José Nicolás F. Frecuencia y desenlace de Balanitis Xerótica Obliterante en pacientes llevados a corrección de Hipospadias. Soc Colomb Urol 2013;37(02):105 